\title{
Universal Forms for One-Dimensional Quantum Hamiltonians: A Comparison of the SUSY and the De La Peña Factorization Approaches
}

\author{
L. Canderle, ${ }^{1}$ A. Plastino,${ }^{2}$ M. Casas, ${ }^{3}$ and A. R. Plastino ${ }^{2,4}$ \\ ${ }^{1}$ Department of Physics, National University of La Pampa, La Pampa, Argentina \\ ${ }^{2}$ National University of La Plata (UNLP), IFLP-CCT-CONICET C. C. 727, 1900 La Plata, Argentina \\ ${ }^{3}$ Departament de Física, IFISC-CSIC, Universitat de les Illes Balears, 07122 Palma de Mallorca, Spain \\ ${ }^{4}$ Instituto Carlos I de Física Teórica y Computacional, Universidad de Granada, 18071 Granada, Spain
}

Correspondence should be addressed to A. Plastino, plastino@fisica.unlp.edu.ar

Received 6 May 2009; Revised 28 June 2009; Accepted 24 August 2009

Recommended by Roger Grimshaw

\begin{abstract}
We show that by linking two factorization techniques often employed to solve Schroedinger's equation one can give any one-dimensional hamiltonian the same form in terms of quantities typical of these approaches. These are the supersymmetric technique (SUSY) and the one of De La Peña's. It is shown that the linkage between them exhibits interesting peculiarities, that are illustrated in the case of a very important family of quantum potentials, namely, reflection-less ones.
\end{abstract}

Copyright $@ 2009$ L. Canderle et al. This is an open access article distributed under the Creative Commons Attribution License, which permits unrestricted use, distribution, and reproduction in any medium, provided the original work is properly cited.

\section{Introduction}

Due to its great importance from the conceptual, practical, and educational viewpoints, the one-dimensional Schroedinger equation (ODSE) has been the subject of continuous attention by researchers since the advent of quantum mechanics in the 1920s. ODSE-interest has been further stimulated because of its relevance in connection with interesting problems in other areas of theoretical physics. One can mention the analysis of exact multisoliton solutions to certain Hamiltonians dynamical systems governed by equations such as the Korteweg-de Vries and sine-Gordon ones [1-5]. The factorization approach [6] is an elegant method to tackle Schroedinger's equation that has been in use for more than half a century (see the excellent review of [7] and references therein). In the last 30 years two important factorization approaches have become popular. One of them is the supersymmetric one (SUSY) [1-5], fully equivalent to the Infeld-Hull-methodology, which adds the notion of 
shape-invariance. The other is due to De La Peña's one [8]. The SUSY approach, in particular, has lead to remarkable progress towards the completion of the program of clasifying all the exactly solvable one-dimensional potentials. The SUSY procedure has also been useful for developing powerful new approximation techniques for solving the Schroedinger equation. We wish to affect here a SUSY-de la Peña comparison and ascertain to what an extent they are equivalent and are able to shed light on each other. The task allows us to give a special universal form to any one-dimensional Hamiltonian. Reference to a specific example helps in such an endeavor, and we will use the one-dimensional well that includes a $\cosh ^{-1}(x)$ squared term, a particularly interesting case of reflection-less potential [9], since it does not reflect waves at any energy.

The paper is organized as follows. We begin our considerations by briefly reviewing the supersymmetric formalism (SUSY) [1-5] in Section 2. Section 3 is a recapitulation of De La Peña's treatment. Our original materials are contained in Section 4, where we link De La Peña's methodology to SUSY. Finally, some conclusions are drawn in Section 5.

\section{The Supersymmetrical Formalism}

The quantum mechanical supersymmetric formalism (SUSY) [1-5] revolves around specific relations between (i) eigenenergies, (ii) eigenfunctions, and (iii) phase shifts with regards to the Hamiltonians

$$
H^{1,2}=-\left[\frac{\hbar^{2}}{2 m}\right]\left[\frac{d^{2}}{d x^{2}}\right]+V^{1,2}
$$

associated with two supersymmetric partner potentials $V^{1}$ and $V^{2}$. The ground state energy of $H^{1}$ is assumed to be zero. The Hamiltonian operators $H^{1,2}$ are factored as $H^{1}=Q^{\dagger} Q$, and $H^{2}=Q Q^{\dagger}$. The operators $Q=(\hbar / \sqrt{2 m})(d / d x)+W$ and $Q^{\dagger}=-(\hbar / \sqrt{2 m})(d / d x)+W$, are given in terms of the so-called quantum superpotential $W(x)$, that, in turn, is related to the two functions $V^{1,2}$ as specified below. The superpotential $W$ can be obtained from the ground state wave function $\psi_{0}$ of $H^{1}$ in the fashion

$$
W(x)=-\left(\frac{\hbar}{\sqrt{2 m}}\right)\left[\frac{1}{\psi_{0}^{(1)}}\right]\left[\frac{d \psi_{0}^{(1)}}{d x}\right] .
$$

The partner Hamiltonians $H^{1}$ and $H^{2}$ possess exactly the same energy spectra, except for the fact that $H^{2}$ has one bound state less than $H^{1}$ [1-5]. The eigen-energies and eigenstates of the partner Hamiltonians $H^{1,2}$ are related in the following manner: $E_{0}^{(1)}=0, E_{n}^{(2)}=E_{n+1}^{(1)}(n=$ $0,1, \ldots)$,

$$
\left(E_{n+1}^{(1)}\right)^{-1 / 2} Q \psi_{n+1}^{(1)}=\psi_{n}^{(2)}, \quad\left(E_{n}^{(2)}\right)^{-1 / 2} Q^{\dagger} \psi_{n}^{(2)}=\psi_{n+1}^{(1)} \quad(n=0,1, \ldots)
$$


The above factorization formalism generalizes that of the harmonic oscillator (HO) Hamiltonian, that introduces the creation-annihilation operators $a$ and $a^{\dagger}$ so as to express it as a product of them. From here on we set $\hbar=m=1$, which redefines $W$ :

$$
W(x)=-\left(\frac{1}{\sqrt{2}}\right)\left[\frac{1}{\psi_{0}^{(1)}}\right]\left[\frac{d \psi_{0}^{(1)}}{d x}\right] .
$$

The two Hamiltonians' spectra can be derived one from the other, which is handy when one of them is exactly solvable. Then, $Q^{ \pm}$reads

$$
Q^{ \pm}=\frac{1}{\sqrt{2}}\left[\mp \frac{d}{d x}+\sqrt{2} W(x)\right]
$$

and one has

$$
V^{1}=\left(-\frac{1}{\sqrt{2}} W^{\prime}+W^{2}\right), \quad V^{2}=\left(\frac{1}{\sqrt{2}} W^{\prime}+W^{2}\right)
$$

that is,

$$
V^{1}+V^{2}=2 W^{2}, \quad V^{2}-V^{1}=\sqrt{2} W^{\prime} .
$$

Also, integration of (2.4) yields

$$
\psi_{0}^{1}=N \exp \left(-\int d x W(x)\right)
$$

$N$ above is a normalization constant. Equation (2.8) can be inverted if a suitable function $W$ is given, which, in turn, would led us to a specific Hamiltonian using the prescription described in the preceding subsection.

\subsection{SUSY and Reflection-Less Potentials}

Reflection-less potentials are those associated with a zero reflection coefficient. Resonant tunnelling phenomena are of great interest. We recapitulate here some well-known results [10-17]. The penetrability coefficient of the pertinent barrier during the resonant tunnelling becomes large to a maximum extent. The attention devoted to reflection-less potentials is due to the fact that they exhibit a penetrability coefficient of almost unity in a whole region of the energy spectrum, whereas resonant tunnelling exists only at selected energy levels. The number of papers devoted to study of properties of the reflection-less quantum systems has been lately increasing. Here we mention just two excellent reviews $[18,19]$ in which one finds both (i) methods for a detailed analysis of the properties of one- and multichannel reflection-less quantum systems, and also (ii) several simple approaches for their qualitative 
understanding. All these methods have found application in scattering theory (direct and inverse). As an interesting illustration consider potentials of the form

$$
V^{1}=\frac{1}{2}\left(n^{2}-\frac{n(n+1)}{\cosh ^{2} x}\right) ; \quad V^{2}=\frac{1}{2}\left(n^{2}-\frac{n(n-1)}{\cosh ^{2} x}\right)
$$

which are supersymmetric partners. In the case $n=1$ (related to the sine-Gordon theory $[20,21])$ we have

$$
V^{2}=\frac{1}{2} \text { (free particle); } \quad V^{1}=\frac{1}{2}\left(1-\frac{2}{\cosh ^{2} x}\right)
$$

We remark that $V^{1}$ is a potential that plays a critical role with regards to soliton-solutions in de Korteweg-de Vries (KdV) hierarchy. With the $n=1$ solutions on can also get the eigenfunctions and eigenvalues for $n=2$ (related to the $\phi^{4}$-theory $[22,23]$ ), and so on. The $V^{1}$-partner is the free-particle Hamiltonian and

$$
\psi_{0}^{1}=\frac{1}{\sqrt{2}} \cosh ^{-1} x
$$

of energy $E_{0}^{1}=0$. In turn, for other potentials of the type (2.9) with $n>1$, that is, for $\psi_{n}^{2}$ and $E_{n}^{2}$, one has

$$
\psi_{n}^{2}=e^{i n x}, \quad E_{n}^{2}=\frac{1}{2}\left(1+n^{2}\right)
$$

while

$$
\psi_{n+1}^{1}=\frac{1}{\sqrt{E_{n}^{2}}} Q^{+} \psi_{n}^{2}=\frac{(\tanh x-i n)}{\sqrt{1+n^{2}}} e^{i n x}
$$

and we see that the states $\psi_{n+1}^{1}$ do not contain reflected waves. The energy eigenvalues are $\mathbf{E}_{n}^{2}$. Successive reiteration of the approach yields the eigenstates of the potentials $n(n-1) / 2 \cosh ^{2} x$ starting from the free-particle instance. The number of bound states is $n$. For the gs one gets, from $(d / d x+n \tanh x) \psi_{0}^{1}=0$,

$$
\psi_{0}^{1}(x) \propto \frac{1}{\cosh ^{2} x}
$$

The gs-energy equals 0 , and the remaining eigenvalues are identical to those for $V^{2}$. Application of $Q_{n}^{+}=(1 / \sqrt{2})(-d / d x+n \tanh x)$ gives the remaining eigenstates. SUSY allows one to relate transmission and reflection coefficients for potentials associated to continuous spectra. Define now

$$
W(x \longrightarrow \pm \infty) \equiv W_{ \pm}
$$


and thus,

$$
V_{1,2} \longrightarrow W_{ \pm}^{2} \text { for } x \longrightarrow \pm \infty
$$

Consider an incoming (from $x \rightarrow-\infty$ ) plane wave $e^{i k x}$ of energy $E$ [11-17]. After $V_{1,2^{-}}$ scattering, we get transmitted waves $T_{1,2}(k) e^{i k^{\prime} x}$ and reflected ones $R_{1,2}(k) e^{-i k x}$, so that [1117]

$$
\begin{gathered}
\psi^{(1,2)}(k, x \longrightarrow-\infty) \longrightarrow e^{i k x}+R_{1,2} e^{-i k x}, \\
\psi^{(1,2)}(k, x \longrightarrow+\infty) \longrightarrow T_{1,2} e^{i k^{\prime} x},
\end{gathered}
$$

with $k$ and $k^{\prime}$ being of the form [11-17]

$$
k=\left(E-W_{-}^{2}\right)^{1 / 2}, \quad k^{\prime}=\left(E-W_{+}^{2}\right)^{1 / 2}
$$

SUSY connects continuous same-energy w.f.s of, respectively, $H_{1}, H_{2}$ as in the discrete instance. One has [11-17]

$$
\begin{aligned}
\psi_{n}^{(2)} & =\left[E_{n+1}^{(1)}\right]^{-1 / 2} Q \psi_{n+1}^{(1)}, \\
\psi_{n+1}^{(1)} & =\left[E_{n}^{(n)}\right]^{-1 / 2} Q^{\dagger} \psi_{n}^{(2)}, \\
e^{i k x}+R_{1} e^{-i k x} & =N\left[\left(-i k+W_{-}\right) e^{i k x}+\left(i k+W_{-}\right) e^{-i k x} R_{2}\right], \\
T_{1} e^{i k^{\prime} x} & =N\left[\left(-i k^{\prime}+W_{+}\right) e^{i k^{\prime} x} T_{2}\right],
\end{aligned}
$$

with $N$ being a normalization constant. Equating same-exponent-terms we find, after elimination of $N$ [11-17]

$$
\begin{aligned}
& R_{1}(k)=\left(\frac{W_{-}+i k}{W_{-}-i k}\right) R_{2}(k), \\
& T_{1}(k)=\left(\frac{W_{+}-i k^{\prime}}{W_{-}-i k}\right) T_{2}(k) .
\end{aligned}
$$

Note that (i) $\left|R_{1}\right|^{2}=\left|R_{2}\right|^{2}$ and also $\left|T_{1}\right|^{2}=\left|T_{2}\right|^{2}$. (ii) $R_{1}\left(T_{1}\right)$ and $R_{2}\left(T_{2}\right)$ share poles in the complex plane, saving for the fact that $R_{1}\left(T_{1}\right)$ has an extra-pole at $k=-i W_{-}$. The pole lies on the positive imaginary axis only if $W_{-}<0$, corresponding to a bound state of null energy. (iii) For $W_{+}=W_{-}$one has $T_{1}(k)=T_{2}(k)$. (iv) For $W_{-}=0, R_{1}(k)=-R_{2}(k)$. If one of the partnerpotentials is a constant (free particle), the the other partner is reflectionless. Consequently, potentials of the type $V(x)=A \sec h^{2} \alpha x$ play a critical role in soliton-studies. 


\section{De La Peña's Treatment}

We pass now to consider the alternative approach of [8]. This is a factorization method that provides an easily implementable algorithm for the purpose, based in the commutator and anticommutator of the creation and destruction operators that affect the factorization. These two operators play here the role of the SUSY-partner potentials. From a practical viewpoint the two techniques are equivalent. Notice that De La Peña's one was advanced one year before Witten's celebrated paper [1-5], so that one cannot ask for it to be superior in any sense to SUSY. As a matter of fact, De La Peña introduced his ideas in a journal that devotes much space to teaching techniques so as to provide beginners with an intuitive grasp of the factorization method (we will follow below the notation of [8]), as applied to any Hermitian operator $\widehat{P}$. His technique is then of utility to anyone without great experience in Quantum Mechanics. He denotes with $\{|n\rangle\}$ its eigenstate-set, a complete basis for Hilbert's space, that for simplicity's sake we assume to be characterized by the single quantum number $n$. Let $\left\{p_{n}\right\}$ be the $\widehat{P}$-spectrum. Then

$$
\widehat{P}|n\rangle=p_{n}|n\rangle .
$$

This operator $\widehat{P}$ is to be later identified with the Hamiltonian of the system under consideration. Creation and destruction operators associated to $\widehat{P}$ are to be denoted with $\hat{\eta}^{\dagger}-\widehat{\eta}$ :

$$
\hat{\eta}^{\dagger}=\sum_{n} C_{n}|n+1\rangle\left\langle n\left|; \quad \hat{\eta}=\sum_{n} C_{n-1}\right| n-1\right\rangle\langle n|,
$$

for as yet unspecified coefficients $C_{n}$ that, when applied to $|n\rangle \equiv|k\rangle$, yield

$$
\hat{\eta}^{\dagger}|k\rangle=C_{k}|k+1\rangle ; \quad \hat{\eta}|k\rangle=C_{k-1}|k-1\rangle .
$$

In determining the coefficients $C_{n}$ De La Peña leaves two of them undetermined, so as to get useful degrees of freedom. The $C_{n}$ are related to the eigenvalues of the bilinear operators $\hat{\eta} \hat{\eta}^{\dagger}$ and $\hat{\eta}^{\dagger} \hat{\eta}$, as seen from (3.3):

$$
\widehat{\eta} \hat{\eta}^{\dagger}|k\rangle=\left|C_{k}\right|^{2}|k\rangle ; \quad \hat{\eta}^{\dagger} \widehat{\eta}|k\rangle=\left|C_{k-1}\right|^{2}|k\rangle \text {. }
$$

Since $|n=0\rangle$ should reasonably be the "vacuum," here

$$
\widehat{\eta}|0\rangle=0 \text {; entailing } C_{-1}=0 \text {. }
$$

Next one considers whether an $n$-upper bound exists or not. If the affirmative is the case, $n=N$, then

$$
\hat{\eta}^{\dagger}|N\rangle=0 \text {; entailing } C_{N}=0 \text {. }
$$


Otherwise, such condition is missing. The degrees of choice mentioned above can be put to good use by requiring $\widehat{P}$ to be of a linear nature

$$
\widehat{P}=a_{00}+a_{10} \widehat{\eta}_{\hat{\eta}}^{\dagger}+a_{01} \hat{\eta}^{\dagger} \widehat{\eta}
$$

With this form for $\widehat{P}$ and (3.5) we evaluate its eigenvalues $p_{m}$ according to

$$
p_{m}=a_{00}+a_{10}\left|C_{m}\right|^{2}+a_{01}\left|C_{m-1}\right|^{2} .
$$

At this stage we are left with $(N+3)$ unknowns, namely, $a_{00}, a_{10}, a_{01}$, and $\left\{\left|C_{n}\right|, n=\right.$ $0,1,2, \ldots, N-1\}$. To determine them we have at our disposal the $N+1$ conditions arising from (3.8), with $n=0,1,2, \ldots, N$, plus normalization, that is, scale-fixing, and its "origin"choice, which suffices for reproducing the $\widehat{P}$-spectrum. We realize now that for representing an arbitrary operator it is easier to work with the product of the operators $\hat{\eta}$ and $\hat{\eta}^{\dagger}$ than with these operators themselves. This forces us to introduce yet two new operators as linear combinations of the just mentioned product:

$$
\widehat{A}=\left[\widehat{\eta}, \hat{\eta}^{\dagger}\right]=\widehat{\eta} \hat{\eta}^{\dagger}-\hat{\eta}^{\dagger} \widehat{\eta} ; \quad \widehat{S}=\left\{\widehat{\eta}, \hat{\eta}^{\dagger}\right\}=\widehat{\eta} \hat{\eta}^{\dagger}+\hat{\eta}^{\dagger} \widehat{\eta},
$$

so that one casts $\widehat{P}$ in a fashion that constitutes the main advantage of the approach

$$
\widehat{P}=q_{0}+q_{a} \widehat{A}+q_{s} \widehat{S}
$$

where the constants in the equation are fitted by comparison with the known form of $\widehat{P}$. The eigenvalues of the just introduced operators $\widehat{A}$ and $\widehat{S}$ are called, respectively, $a_{k}$ and $s_{k}$ and are obtained via (3.3) and (3.9):

$$
\widehat{A}|k\rangle=a_{k}|k\rangle=\left(\left|C_{k}\right|^{2}-\left|C_{k-1}\right|^{2}\right)|k\rangle ; \quad \widehat{S}|k\rangle=s_{k}|k\rangle=\left(\left|C_{k}\right|^{2}+\left|C_{k-1}\right|^{2}\right)|k\rangle,
$$

entailing

$$
a_{k}=\left|C_{k}\right|^{2}-\left|C_{k-1}\right|^{2} ; \quad s_{k}=\left|C_{k}\right|^{2}+\left|C_{k-1}\right|^{2}
$$

which in turn are manipulated to yield, after summation and subtraction of them,

$$
\left|C_{k}\right|^{2}=\frac{1}{2}\left(s_{k}+a_{k}\right)=\frac{1}{2}\left(s_{k+1}-a_{k+1}\right) .
$$

With these results one ascertains that the spectra of $\widehat{A}-\widehat{S}$ fulfill a set of consistency relations. We get, (i) from (3.12) and (3.13),

$$
s_{k} \geq 0 ; \quad s_{k}+a_{k} \geq 0 ; \quad s_{k}-a_{k} \geq 0
$$


further (ii) from (3.13),

$$
s_{k+1}-s_{k}=a_{k+1}+a_{k}
$$

and (iii), from (3.12) and (3.6),

$$
s_{0}=a_{0} ; \quad s_{N}=-a_{N} .
$$

These relations can be interpreted as follows. Equations (3.14) show that the spectrum of $\widehat{S}$ is nonnegative and with lower bound $a_{0}$. The basic condition (3.15) fixes the $\widehat{P}_{\text {- }}$ spectrum's structure. Lower and (possible) upper spectral bounds are determined by (3.16). If application of (3.16) leads to a contradiction, this entails that no upper bound exists. The consistency relations (3.14), (3.15), and (3.16) contain all possible information concerning $\widehat{P}$ spectrum in a most compact and accessible fashion, given that one knows the operators $\widehat{A}$ and $\widehat{S}$ associated to any $\widehat{P}$.

\section{Relation between SUSY and De La Peña}

As recounted above, De La Peña introduces his two operators $\widehat{\eta}, \hat{\eta}^{\dagger}[8]$ so as to be in a position to cast his all-important operator in the bilinear fashion (3.7) and then employs two auxiliary operators $\widehat{A}, \widehat{S}$ (3.9) so as to cast $\widehat{P}$ in the final form (3.10). It seems rather natural to identify with each other the creation-annihilation operators of the two factorization approaches, that is, SUSY's $\widehat{Q}^{-}, \widehat{Q}^{+}$with, respectively, $\widehat{\eta}$ and $\widehat{\eta}^{\dagger}$. Such a procedure then tells us that

$$
\begin{aligned}
\widehat{A} \psi(x) & =\widehat{Q}^{-} \widehat{Q}^{+} \psi-\widehat{Q}^{+} \widehat{Q}^{-} \psi \\
& =\frac{1}{2}\left(\frac{d}{d x}+\sqrt{2} W\right)\left(-\frac{d}{d x}+\sqrt{2} W\right) \psi-\frac{1}{2}\left(-\frac{d}{d x}+\sqrt{2} W\right)\left(\frac{d}{d x}+\sqrt{2} W\right) \psi,
\end{aligned}
$$

which, after suitable manipulations, leads to

$$
\widehat{A} \psi(x)=\sqrt{2} W^{\prime} \psi(x)
$$

entailing

$$
\widehat{A}=\sqrt{2} W^{\prime} \widehat{\mathbf{1}},
$$

involving the unity operator.

This is an important relation, our first new result, linking the De La Peña operator $\widehat{A}$ to the SUSY superpotential. It generalizes the HO-commutation rule, in which $\left[\widehat{a}, \widehat{a}^{\dagger}\right]=1$. In other words, De La Peña's $\widehat{A}$ carries information regarding the way the SUSY's $\widehat{Q}^{ \pm}$differ 
from their HO-counterparts. The $\widehat{Q}^{ \pm}$commutators become now nontrivial operators instead of the customary, HO-related constants. Analogously (cf. (2.2)),

$$
\widehat{S} \psi(x)=\widehat{Q}^{-} \widehat{Q}^{+} \psi+\widehat{Q}^{+} \widehat{Q}^{-} \psi=-\frac{d^{2} \psi}{d x^{2}}+2 W^{2} \psi \Longrightarrow \widehat{S}=-\frac{d^{2}}{d x^{2}}+2 W^{2}=2\left[-\left(\frac{1}{2}\right) \frac{d^{2}}{d x^{2}}+W^{2}\right] .
$$

Notice that $\widehat{S}$ is proportional to a Hamiltonian form in which the square of $W$ acts as the potential. More specifically, De La Peña's $\widehat{S}$ is the Hamiltonian of a particle of mass $4 \mathrm{~m}$ (cf. (2.1)) moving in a $2 W^{2}$-well.

We reach thus an interesting conclusion from the present linkage between two factorization methods: De La Peña's way of splitting its operator $\widehat{P}$, to be later identified with the system's Hamiltonian $H^{1}$, shows that it has a pure "Hamiltonian" component plus one that relates to the form in which the supersymmetric $Q^{ \pm}$differ from their HO-counterparts. Combining now (2.7), (4.3), and (4.4) leads immediately to

$$
\widehat{S}=\widehat{H}^{1}+\widehat{H}^{2} ; \quad \widehat{A}=\widehat{V}^{2}-\widehat{V}^{1}=\widehat{H}^{2}-\widehat{H}^{1},
$$

which entails expressing De La Peña's all important operator $\widehat{P}$ in SUSY terms

$$
\begin{aligned}
\widehat{P} & =q_{0}+q_{a} \widehat{A}+q_{s} \widehat{S}=q_{0}+q_{a}\left(\widehat{H}^{2}-\widehat{H}^{1}\right)+q_{s}\left(\widehat{H}^{2}+\widehat{H}^{1}\right) \\
& =q_{0}+2 q_{s}\left[K+W^{2}\right]+\sqrt{2} q_{a} W^{\prime} \\
& =q_{0}+2 q_{s} \widehat{H}^{S}+\sqrt{2} q_{a} W^{\prime},
\end{aligned}
$$

where $\widehat{H}^{S}$ is a putative Hamiltonian whose potential is the square of the superpotential. We immediately realize that De La Peña's approach seems to require some additional information vis-a-vis SUSY, namely, the three parameters $q_{0}, q_{s}, q_{a}$, which might, at first sight, be regarded as superfluous information, since SUSY (a complete treatment) does not need them. This is not so, as the following example will show.

\subsection{Application of the De La Peña-SUSY Linkage}

As an application we tackle the potentials discussed in Secion 1. In dealing with a potential of the form

$$
V^{1}=\frac{1}{2}\left[1-\frac{2}{\cosh ^{2} x}\right]
$$

that is derived from the general instance

$$
V^{1}=\frac{1}{2}\left[n^{2}-\frac{n(n+1)}{\cosh ^{2} x}\right]
$$


for $n=1$ we are left with a Hamiltonian

$$
\widehat{H}^{1} \equiv \widehat{H}=\frac{p^{2}}{2}+\frac{1}{2}\left[1-\frac{2}{\cosh ^{2} x}\right]=\frac{p^{2}}{2}-\frac{1}{\cosh ^{2} x}+\frac{1}{2} .
$$

Here we must identify De La Peña's $\widehat{P}$ with the Hamiltonian. We have

$$
\begin{gathered}
H^{2}=\frac{p^{2}}{2}+\frac{1}{2} \text { entailing } \widehat{S}=H^{1}+H^{2}=p^{2}+1-\operatorname{sech}^{2}(x), \\
\widehat{A}=H^{2}-H^{1}=\operatorname{sech}^{2}(x),
\end{gathered}
$$

which agrees nicely with (4.3).

Thus, looking back at (3.10)

$$
\widehat{H}=q_{0}+q_{s} \widehat{S}+q_{a} \widehat{A}=q_{0}+q_{s}\left[p^{2}+1-\operatorname{sech}^{2}(x)\right]+q_{a} \operatorname{sech}^{2}(x)
$$

we can determine the values of the constants $q_{0}, q_{a}, q_{s}$ that relate $\widehat{P} \equiv \widehat{H}$ to $\widehat{A}$ and $\widehat{S}$. We find $q_{s}=1 / 2, q_{a}=-1 / 2$, and $q_{0}=0$. Consequently, we get the "correct" spectrum.

\section{Conclusions}

We have compared here two factorization methods in vogue lately, namely, SUSY and De La Peña's (the S-DLP linkage). We were able to establish a relation between them that yields some new insight into supersymmetry. Given a Hamiltonian $\widehat{H}$ that is to be factorized via the creation-destruction operators $\widehat{Q}^{ \pm}$, the above linkage shows that $\widehat{H}$ gets partitioned into a linear combination of two DLP-terms, involving the DLP-operators $\widehat{S}$ and $\widehat{A}$. The first one has the Hamiltonian aspect, but with the square of the superpotential as a pseudopotential, while $\widehat{A}$ refers to the way in which the $\widehat{Q}^{ \pm}$differ from HO's creation-destruction operators. We also see that the commutator of the supersymmetric creation-destruction operators becomes a nontrivial operator, linked to De La Peña's operator $\widehat{A}$. Summing up, we have shown that by linking two factorization techniques one can give any one-dimensional Hamiltonian the same form in terms of quantities typical of these approaches.

\section{Acknowledgment}

This work was partially supported by the MEC Grant FIS2008-00781 (Spain) and by the Projects FQM-2445 and FQM-207 of the Junta de Andalucia (Spain, EU).

\section{References}

[1] F. Cooper, A. Khare, and U. Sukhatme, Supersymmetry in Quantum Mechanics, World Scientific, River Edge, NJ, USA, 2001.

[2] F. Cooper, A. Khare, and U. Sukhatme, "Supersymmetry and quantum mechanics," Physics Reports, vol. 251, no. 5-6, pp. 267-385, 1995. 
[3] B. K. Bagchi, Supersymmetry in Quantum and Classical Mechanics, vol. 116, Chapman \& Hall/CRC, Boca Raton, Fla, USA, 2001.

[4] G. Junker, Supersymmetric Methods in Quantum and Statistical Physics, Texts and Monographs in Physics, Springer, Berlin, Germany, 1996.

[5] E. Witten, "Search for a realistic Kaluza-Klein theory," Nuclear Physics. B, vol. 186, no. 3, pp. 412-428, 1981.

[6] L. Infeld and T. E. Hull, "The factorization method," Reviews of Modern Physics, vol. 23, no. 1, pp. 21-68, 1951.

[7] J. Oscar OrtizA. Ballesteros, et al., "On the factorization method in quantum mechanics," p. 285, University of Burgos.

[8] L. de la Peña and R. Montemayor, "Raising and lowering operators and spectral structure: a concise algebraic technique," American Journal of Physics, vol. 48, no. 10, pp. 855-860, 1980.

[9] J. Lekner, "Reflectionless eigenstates of the sech ${ }^{2}$ potential," American Journal of Physics, vol. 75, no. 12, pp. 1151-1157.

[10] N. Saito and Y. Kayanuma, "Resonant tunnelling of a composite particle through a single potential barrier," Journal of Physics: Condensed Matter, vol. 6, no. 20, pp. 3759-3766, 1994.

[11] D. Boyanovsky and R. Blankenbecler, "Fractional indices in supersymmetric theories," Physical Review D, vol. 30, no. 8, pp. 1821-1824, 1984.

[12] R. Akhoury and A. Comtet, "Anomalous behavior of the Witten index-exactly soluble models," Nuclear Physics B, vol. 246, no. 2, pp. 253-278, 1984.

[13] C. V. Sukumar, "Supersymmetry, potentials with bound states at arbitrary energies and multisoliton configurations," Journal of Physics A, vol. 19, no. 12, pp. 2297-2316, 1986.

[14] D. Baye, "Supersymmetry between deep and shallow nucleus-nucleus potentials," Physical Review Letters, vol. 58, no. 26, pp. 2738-2741, 1987.

[15] F. Cooper, J. N. Ginocchio, and A. Wipf, "Derivation of the $S$-matrix using supersymmetry," Physics Letters A, vol. 129, no. 3, pp. 145-147, 1988.

[16] R. D. Amado, F. Cannata, and J.-P. Dedonder, "Supersymmetric quantum mechanics, phase equivalence, and low energy scattering anomalies," Physical Review C, vol. 43, no. 5, pp. 2077-2081, 1991.

[17] A. A. Andrianov, M. V. Ioffe, F. Cannata, and J.-P. Dedonder, "Second order derivative supersymmetry, $q$ deformations and the scattering problem," International Journal of Modern Physics A, vol. 10, no. 18, pp. 2683-2702, 1995.

[18] B. N. Zakhariev and V. M. Chabanov, Physics of Elementary Particles and Atomic Nuclei, vol. 25, pp. 1561-1597, 1994.

[19] B. N. Zakhariev and V. M. Chabanov, "The qualitative theory of elementary transformations of one- and multichannel quantum systems in the inverse-problem approach. The construction of transformations with given spectral parameters," Physics of Particles and Nuclei, vol. 30, no. 2, pp. 111-130, 1999.

[20] A. Barone, F. Esposito, C. J. Magee, and A. C. Scott, "Theory and applications of the sine-gordon equation," La Rivista del Nuovo Cimento, vol. 1, no. 2, pp. 227-267, 1971.

[21] S. Homma and S. Takeno, "Numerical evidence of the existence of approximate Kink-Antikink states and breather-like modes in sine-form discrete double sine-Gordon equation," Progress of Theoretical Physics, vol. 74, no. 3, pp. 618-621, 1985.

[22] J. Rubinstein, "Sine-Gordon equation," Journal of Mathematical Physics, vol. 11, no. 1, pp. 258-266, 1971.

[23] R. Rajaraman, "Some non-perturbative semi-classical methods in quantum field theory (a pedagogical review)," Physics Reports, vol. 21, no. 5, pp. 227-313, 1975. 


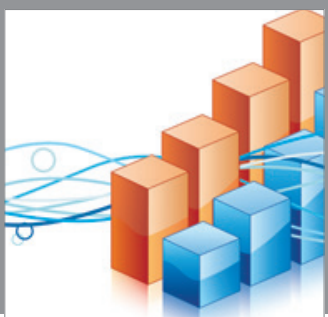

Advances in

Operations Research

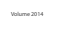

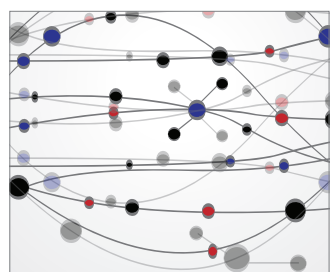

\section{The Scientific} World Journal
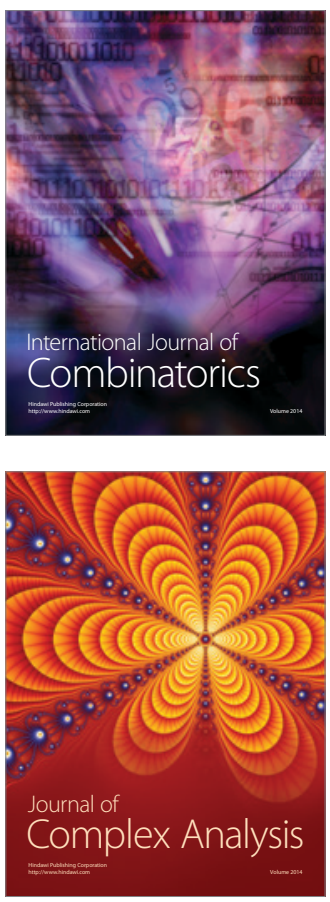

International Journal of

Mathematics and

Mathematical

Sciences
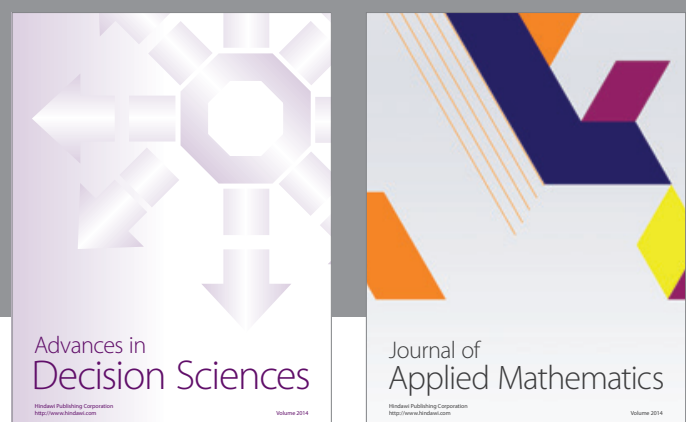

Journal of

Applied Mathematics
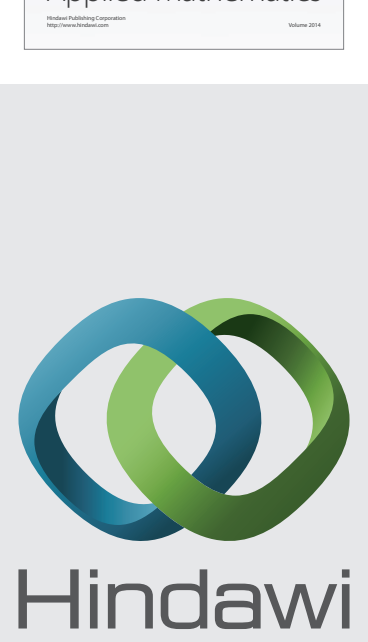

Submit your manuscripts at http://www.hindawi.com
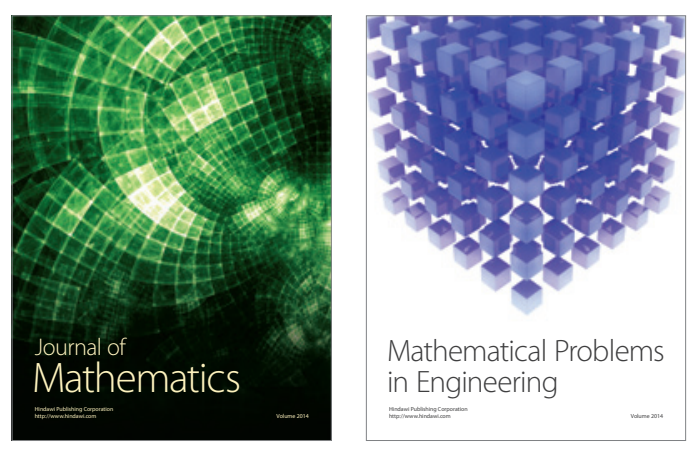

Mathematical Problems in Engineering
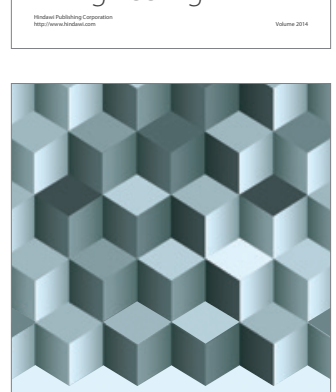

Journal of

Function Spaces
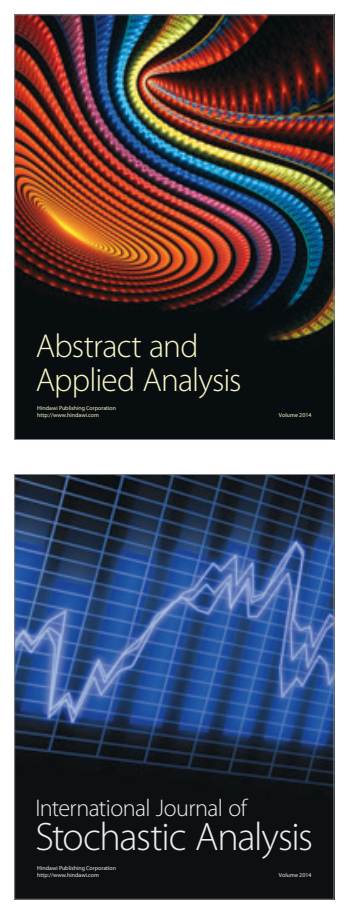

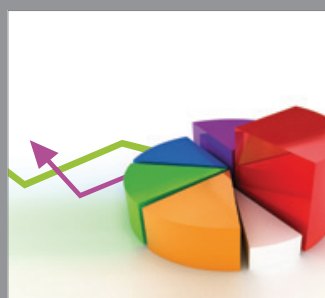

ournal of

Probability and Statistics

Promensencen
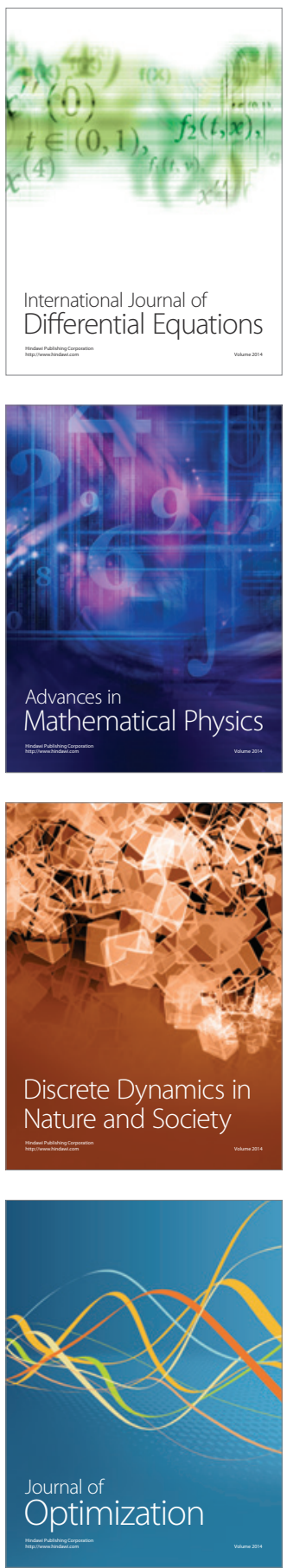